\title{
Independent Sets in Corona Graphs
}

\author{
Carmen Ortiz , Mònica Villanueva ${ }^{(0)}$
}

\begin{abstract}
The corona of two graphs $G$ and $H$ is the graph $G \odot H$ obtained by taking one copy of $G ;|V(G)|$ copies of $H$; and joining each vertex of the $i$-th copy of $H$ to the $i$-th vertex of $G$. For general graphs, counting independent sets as well as maximal independent sets are $\# \mathcal{P}$ - complete problems. In this work, for a general graph $H$ we determine the number of independent sets in $K_{n} \odot H, K_{1, n} \odot H$, $\overline{K_{n}} \odot H$ and $W_{1, n} \odot H$. We also establish the number of maximal independent sets of the corona graph $G \odot H$ of two general graphs $G$ and $H$.
\end{abstract}

\section{Introduction}

$G=(V, E)$ is a finite undirected connected graph with no multiple edges or self loops, vertex-set $V(G)=V$ and edge-set $E(G)=E$. The neighbourhood of a vertex $v$ in $G$ is $N[v]=\{u \in V \mid(u, v) \in E\} \cup\{v\}$. For a positive integer $n$, the complete graph, the chordless path and the chordless cycle on $n$ vertices are denoted by $K_{n}, P_{n}$ and $C_{n}$, respectively. $K_{1, n}$ is the star tree with $n$ pendant vertices. The wheel graph $W_{1, n}$ has a single vertex $u$ connected to all vertices of an $n$-cycle.

2000 AMS Subject Classification: 05C69, 05C76.

Keywords and Phrases: independent sets, corona graphs. 
A clique in a graph $G$ is a maximal complete subgraph of $G$. An independent set of $G$ is a subset $S$ of $V$ such that no two vertices are adjacent. $S \subseteq V$ is a maximal independent set (mis) if it is not properly contained in any other independent set of $G$. The number of mis of a graph $G$ is $\mu(G)$. An independent set is maximum if it has maximum cardinality. The size of a maximum independent set of $G$ is denoted by $\alpha(G)$.

A molecule can be modeled as a graph with vertices representing atoms and edges representing bonds. A topological index is associated with chemical compounds to predict some properties since there is a very close relation between chemical characteristics of many compounds and the topological structure of its molecular graph. The Merrifield-Simmons index is the number of independent sets of the associated molecular graph and it is correlated with the boiling point of the molecule [9].

The molecular graph of some chemical compounds is obtained as a corona graph. For example, cycloalkanes with a single ring [17]. Various topological indices of different corona graphs have been studied. The Merrifield-Simmons index of a caterpillar graph $P_{n} \odot K_{1}$ and a sunlet graph $C_{n} \odot K_{1}$ are determined by Reyhani et al. [14]. Wu et al. [16] studied this index for the corona graphs $P_{n} \odot K_{2}$ and $C_{n} \odot K_{2}$. Hamzed et al. [6] discussed the cases $C_{n} \odot H$ and $P_{n} \odot H$.

A Clar structure is a mis of the Clar graph of the corresponding benzenoid hydrocarbons [3]. This paper deals with benzenoid hydrocarbons whose Clar graphs are either paths or cycles.

Valiant [15] showed that the problem of counting the number of mis is $\# \mathcal{P}$ - complete for a general graph. Okamoto et al. [10] proved that the problem remains so even for chordal graphs. Li et al. [8] determined the largest number of mis among all $n$-vertex bipartite graphs with at least one cycle. Hujter and Tuza [7] and Chang and Jou [1] solved the problem for triangle-free graphs. Ortiz and Villanueva determined $\mu(G)$ of a caterpillar graph [11] and also of grid graphs [12].

In this work, we determine the number of independent sets of various corona graphs: $K_{n} \odot H, K_{1, n} \odot H, \overline{K_{n}} \odot H$ and $W_{1, n} \odot H$ where $H$ is an 
arbitrary graph. We establish the number of mis of $G \odot H$ as a function of the number of independent sets of $G$ and the number of mis of $H$. In the special case of $C_{n} \odot K_{1}$ we show that its number of mis is given by the Fibonacci sequence. We build the independent graph (intersection graph of mis) and the clique graph (intersection graph of cliques) of $C_{n} \odot K_{1}$.

\section{Preliminaries}

The Fibonacci sequence is given by $F_{0}=0, F_{1}=1$ and the recurrence formula $F_{n}=F_{n-1}+F_{n-2}$ for $n \geq 2$. The Lucas sequence is defined by $L_{0}=2, L_{1}=1$ and $L_{n}=L_{n-1}+L_{n-2}$ for $n \geq 2$.

Prodinger and Tichy 13 introduced the Fibonacci number $f(G)$ of a graph $G$ as the number of independent sets of $G$, not necessarily maximal, including the empty set. They proved the following results.

Lemma 2.1. The Fibonacci number of

a) a complete graph is $f\left(K_{n}\right)=n+1$ for $n \geq 1$,

b) a chordless path is $f\left(P_{n}\right)=F_{n+2}$ for $n \geq 1$,

c) a chordless cycle is $f\left(C_{n}\right)=L_{n}$ for $n \geq 3$,

d) a star tree is $f\left(K_{1, n}\right)=2^{n}+1$ for $n \geq 1$,

e) a wheel graph is $f\left(W_{1, n}\right)=f\left(C_{n}\right)+1$ for $n \geq 3$.

Let $f_{k}(G)$ be the number of independent sets of size $k$ of the graph $G$. We define $f_{0}(G)=1$.

Lemma 2.2. a) $f_{1}\left(K_{n}\right)=n$ and $f_{k}\left(K_{n}\right)=0$ with $n \geq 1$ and $k \geq 2$, b) $f_{k}\left(\overline{K_{n}}\right)=\left(\begin{array}{l}n \\ k\end{array}\right)$ with $1 \leq k \leq n$,

c) $f_{k}\left(K_{1, n}\right)=\left(\begin{array}{l}n \\ k\end{array}\right)$ for $2 \leq k \leq n$ and $f_{1}\left(K_{1, n}\right)=n+1$,

d) $f_{1}\left(W_{1, n}\right)=1+f_{1}\left(C_{n}\right)$ and $f_{k}\left(W_{1, n}\right)=f_{k}\left(C_{n}\right)$ for $2 \leq k \leq\left\lfloor\frac{n}{2}\right\rfloor$.

Lemma 2.3. For $n \geq 2$ and $1 \leq k \leq\left\lceil\frac{n}{2}\right\rceil$,

$f_{k}\left(P_{n}\right)=f_{k}\left(P_{n-1}\right)+f_{k-1}\left(P_{n-2}\right)$ with $f_{0}\left(P_{n}\right)=1, f_{1}\left(P_{n}\right)=n$, $f_{2}\left(P_{n}\right)=\left(\begin{array}{c}n-1 \\ 2\end{array}\right), f_{1}\left(P_{1}\right)=1, f_{1}\left(P_{2}\right)=2$ and $f_{2}\left(P_{2}\right)=0$. 
Proof. Let $V\left(P_{n}\right)=\left\{v_{1}, v_{2}, \ldots, v_{a}, \ldots, v_{b}, \ldots, v_{n}\right\}$. If $k=1$, we have $n$ alternatives for choosing one vertex of $P_{n}$.

If $k=2$, let $S=\left\{v_{a}, v_{b}\right\}$ with $1 \leq a \leq b-2 \leq n-2$, be an independent set of $P_{n}$. Since $v_{a}$ can be chosen in $n-2$ different ways then $v_{b}$ has $n-(a+1)$ options. Thus, $f_{2}\left(P_{n}\right)=\sum_{a=1}^{n-2}(n-(a+1))=\frac{1}{2}(n-1)(n-2)$.

For $k \geq 3$, let $S$ be an independent set of $P_{n-1}$ having $k$ vertices. $S$ is also an independent set of $P_{n}$. If we consider an independent set $R$ of $P_{n-2}$ that has $k-1$ vertices, $R \cup\left\{v_{n}\right\}$ is an independent set of $P_{n}$.

Lemma 2.4. For $n \geq 3$ and $1 \leq k \leq\left\lceil\frac{n}{2}\right\rceil, f_{k}\left(P_{n}\right)=\left(\begin{array}{c}n-(k-1) \\ k\end{array}\right)$.

Proof. Applying induction on $n$ we have that $f_{k}\left(P_{n-1}\right)=\left(\begin{array}{c}n-1-(k-1) \\ k\end{array}\right)=$ $\left(\begin{array}{c}n-k \\ k\end{array}\right)$ and $f_{k-1}\left(P_{n-2}\right)=\left(\begin{array}{c}n-2-(k-2) \\ k-1\end{array}\right)=\left(\begin{array}{c}n-k \\ k-1\end{array}\right)$. By binomial coefficient property and Lemma 2.3 , the result follows.

Lemma 2.5. For $n \geq 3$ and $1 \leq k \leq\left\lfloor\frac{n}{2}\right\rfloor, f_{k}\left(C_{n}\right)=f_{k}\left(C_{n-1}\right)+$ $f_{k-1}\left(C_{n-2}\right)$ with $f_{0}\left(C_{n}\right)=1, f_{1}\left(C_{n}\right)=n$ and $f_{2}\left(C_{n}\right)=\frac{n(n-3)}{2}$.

Proof. Let $V\left(C_{n}\right)=\left\{v_{1}, v_{2}, \ldots, v_{n}\right\}$. If $k=1$, we have $n$ alternatives for choosing one vertex of $C_{n}$.

If $k=2$, let $S=\left\{v_{a}, v_{b}\right\}$ be an independent set of $C_{n}$ with $a+1<b$. We have that $v_{a}$ can be either $v_{1}$ or $v_{2}, \ldots$, or $v_{n-2}$. If $a=1$ then $v_{b}$ has $n-3$ options but if $a \neq 1$ then $v_{b}$ has $n-(a+1)$ options. Thus, $f_{2}\left(C_{n}\right)=(n-3)+\sum_{a=2}^{n-2}(n-(a+1))=\frac{n(n-3)}{2}$.

For $k \geq 3$, let $S$ be an independent set of $C_{n-1}$ having $k$ vertices. We have that $S$ is also an independent set of $C_{n}$. Now consider an independent set $R$ in $C_{n-2}$ that has $k-1$ vertices. There are three cases:

-If $v_{1} \in R$ and $v_{n-2} \notin R$ then $R \cup\left\{v_{n-1}\right\}$ is an independent set of $C_{n}$. -If $v_{1} \notin R$ and $v_{n-2} \in R$ then $R \cup\left\{v_{n}\right\}$ is an independent set of $C_{n}$. -If $v_{1} \notin R$ and $v_{n-2} \notin R$ then $R \cup\left\{v_{n}\right\}$ is an independent set of $C_{n}$. In this case $R \cup\left\{v_{n-1}\right\}$ is also an independent set of $C_{n-1}$ and of $C_{n}$. But it has already been considered in $f_{k}\left(C_{n-1}\right)$. 
Lemma 2.6. For $n \geq 3$ and $1 \leq k \leq\left\lfloor\frac{n}{2}\right\rfloor, f_{k}\left(C_{n}\right)=\left(\begin{array}{c}n-k) \\ k\end{array}\right)+\left(\begin{array}{c}n-(k+1) \\ k-1\end{array}\right)$.

Proof. Analogous to the proof of Lemma 2.4.

Lemma 2.7. The number of maximal independent sets of

a) a complete graph is $\mu\left(K_{n}\right)=n$ for $n \geq 1$,

b) [5] a path graph is $\mu\left(P_{n}\right)=\mu\left(P_{n-2}\right)+\mu\left(P_{n-3}\right)$ for $n \geq 4$ with $\mu\left(P_{1}\right)=1$ and $\mu\left(P_{2}\right)=\mu\left(P_{3}\right)=2$,

c) [5] a cycle graph is $\mu\left(C_{n}\right)=\mu\left(C_{n-2}\right)+\mu\left(C_{n-3}\right)$ for $n \geq 6$ with $\mu\left(C_{3}\right)=3, \mu\left(C_{4}\right)=2$ and $\mu\left(C_{5}\right)=5$,

d) the complement of a complete graph $\mu\left(\overline{K_{n}}\right)=1$ for $n \geq 1$,

e) a star tree is $\mu\left(K_{1, n}\right)=2$ for $n \geq 1$,

f) a wheel graph is $\mu\left(W_{1, n}\right)=\mu\left(C_{n}\right)+1$.

\section{Independent Sets in Corona Graphs}

Frucht and Harary [4] defined the corona of two graphs $G$ and $H$ as the graph $G \odot H$ obtained by taking one copy of $G ;|V(G)|$ copies of $H$ and joining the $i$-th vertex of $G$ to every vertex in the $i$-th copy of $H$.

Lemma $3.1([2])$.

a) For two disjoint graphs $G$ and $H: \quad f(G \cup H)=f(G) \cdot f(H)$.

b) For a graph $G$ and a vertex $v \in V(G): f(G)=f(G-v)+f(G-N[v])$.

Theorem 3.1. Let $H$ be an arbitrary graph. The number of independent sets of the graph $K_{n} \odot H$ is:

$$
f\left(K_{n} \odot H\right)=f(H)^{n-1}[f(H)+n] .
$$

Proof. Let $G_{n}=K_{n} \odot H$ and $V\left(K_{n}\right)=\left\{v_{1}, v_{2}, \ldots, v_{n}\right\}$. Applying Lemma 3.1 to $G_{n}$, we have that $G_{n}-v_{n}=G_{n-1} \cup H^{n}$ and $G_{n}-N\left[v_{n}\right]=\cup_{i=1}^{n-1} H^{i}$ where $H^{i}$ is the i-th copy of $H$, with $i=1, \ldots, n$.

Thus $f\left(G_{n}\right)=f(H) f\left(G_{n-1}\right)+f(H)^{n-1}$ and the result follows. 
Theorem 3.2. The number of independent sets of the graph $\overline{K_{n}} \odot H$ is $f\left(\overline{K_{n}} \odot H\right)=(f(H)+1)^{n}$.

Proof. Direct consequence of Lemma 3.1.

Theorem 3.3. The number of independent sets of the graph $K_{1, n} \odot H$ is:

$$
f\left(K_{1, n} \odot H\right)=f(H)^{n}+f(H)(f(H)+1)^{n} .
$$

Proof. Let $G_{n}=K_{1, n} \odot H$ and $V\left(K_{1, n}\right)=\left\{u, v_{1}, v_{2}, \ldots, v_{n}\right\}$. Applying Lemma 3.1 to $G_{n}$ we have that $G_{n}-v_{n}=G_{n-1} \cup H^{n}$ and $G_{n}-N\left[v_{n}\right]=$ $H^{u} \cup\left[\cup_{i=1}^{n-1}\left(v_{i} \odot H^{i}\right)\right]$ where $H^{i}$ is the i-th copy of $H$ and $H^{u}$ is the copy of $H$ connected to the universal vertex $u$.

Thus, $f\left(G_{n}\right)=f(H) f\left(G_{n-1}\right)+f(H)(1+f(H))^{n-1}$. This is equivalent to the recurrence equation: $X_{n}=k X_{n-1}+k(k+1)^{n-1}$ whose solution is $X_{n}=C k^{n-1}+k\left[(k+1)^{n}-k^{n}\right]$ where $k=f(H)$ and $C=k(k+1)$.

Theorem 3.4. The number of independent sets of $G_{n}=W_{1, n} \odot H$ is:

$$
f\left(W_{1, n} \odot H\right)=\left[f\left(C_{n} \odot H\right)\right] f(H)+f(H)^{n} .
$$

Proof. Let $u$ be the universal vertex of $W_{1, n}$. By Lemma 3.1 we have that $G_{n}-u=\left(C_{n} \odot H\right) \cup H$ and $G_{n}-N[u]=H^{1} \cup H^{2} \ldots \cup H^{n}$. Therefore, the result follows.

Hamzed et al. [6] proved that

$$
f\left(C_{n} \odot H\right)=f\left(P_{n-1} \odot H\right) f(H)+f\left(P_{n-3} \odot H\right) f(H)^{2} .
$$

\section{Maximal Independent Sets in Corona Graphs}

Theorem 4.1. Let $|V(G)|=n$ and $|V(H)|=m$. The number of mis of the graph $G \odot H$ is:

$$
\mu(G \odot H)=\sum_{k=0}^{\alpha(G)} f_{k}(G) \mu(H)^{n-k} .
$$

Proof. Let $S$ be an independent set of size $k$ in $G$. For each vertex $v_{i}$ in $G$ such that $v_{i} \notin S$ consider a mis $T^{i}$ of $H^{i}$. We have that $T=\bigcup_{\substack{i=1 \\ v_{i} \notin S}}^{n} T^{i}$ is 
an independent set of $G \odot H$ because $T^{i} \cap T^{j}=\phi$ for $i \neq j$. Thus, $S \cup T$ is also an independent set in $G \odot H$. Moreover, $S \cup T$ is maximal. In fact, if we add a vertex $v_{j} \in V(G) \backslash S$ to $S \cup T$ then it is not an independent set since $v_{j}$ is adjacent to every vertex of $T^{j} \subset T$. On the other hand, if we add a vertex $x \in H^{i}$ for some $i \in\{1,2, \ldots, n\}$ such that $v_{i} \notin S$ then $T^{i}$ is not maximal in $H^{i}$, a contradiction.

Since $H$ has $\mu(H)$ mis and $S$ has size $k$, there are $\mu(H)^{n-k}$ different sets that can be added to $S$. Furthermore, a set that contains one mis of every copy $H^{i}$ is also a mis of $G \odot H$.

Table 1 summarizes the number of mis for some particular cases.

\begin{tabular}{|l||l|l|l|}
\hline$G \backslash H$ & \multicolumn{1}{|c|}{$K_{m}$} & \multicolumn{1}{c|}{$P_{m}$} & \multicolumn{1}{c|}{$C_{m}$} \\
\hline \hline$K_{n}$ & $(n+m) m^{n-1}$ & $\left(n+\mu\left(P_{m}\right)\right) \mu\left(P_{m}\right)^{n-1}$ & $\left(n+\mu\left(C_{m}\right)\right) \mu\left(C_{m}\right)^{n-1}$ \\
\hline$P_{n}$ & $\sum_{k=0}^{\left\lceil\frac{n}{2}\right\rceil} f_{k}\left(P_{n}\right) m^{n-k}$ & $\sum_{k=0}^{\left\lceil\frac{n}{2}\right\rceil} f_{k}\left(P_{n}\right) \mu\left(P_{m}\right)^{n-k}$ & $\sum_{k=0}^{\left\lceil\frac{n}{2}\right\rceil} f_{k}\left(P_{n}\right) \mu\left(C_{m}\right)^{n-k}$ \\
\hline$C_{n}$ & $\sum_{k=0}^{\left\lfloor\frac{n}{2}\right\rfloor} f_{k}\left(C_{n}\right) m^{n-k}$ & $\sum_{k=0}^{\left\lfloor\frac{n}{2}\right\rfloor} f_{k}\left(C_{n}\right) \mu\left(P_{m}\right)^{n-k}$ & $\sum_{k=0}^{\left\lfloor\frac{n}{2}\right\rfloor} f_{k}\left(C_{n}\right) \mu\left(C_{m}\right)^{n-k}$ \\
\hline$K_{1, n}$ & $m^{n}+\sum_{k=0}^{n}\left(\begin{array}{l}n \\
k\end{array}\right) m^{n-k+1}$ & $\mu\left(P_{m}\right)^{n}+\sum_{k=0}^{n}\left(\begin{array}{l}n \\
k\end{array}\right) \mu\left(P_{m}\right)^{n-k+1}$ & $\mu\left(C_{m}\right)^{n}+\sum_{k=0}^{n}\left(\begin{array}{l}n \\
k\end{array}\right) \mu\left(C_{m}\right)^{n-k+1}$ \\
\hline
\end{tabular}

Table 1: Number of Maximal Independent Sets of Corona Graphs.

In [11] it was proven that for $n \geq 1: \mu\left(P_{n} \odot \overline{K_{m}}\right)=F_{n+2}$ with $\mu\left(P_{1} \odot \overline{K_{m}}\right)=2$ and $\mu\left(P_{2} \odot \overline{K_{m}}\right)=3$.

The number of mis of $P_{n} \odot K_{m}$ satisfies $\mu\left(P_{n} \odot K_{m}\right)=m\left[\mu\left(P_{n-1} \odot K_{m}\right)+\mu\left(P_{n-2} \odot K_{m}\right)\right]$ for $m \geq 2 ; n \geq 3$.

\section{Independent and Clique Graph of $G_{n}=C_{n} \odot K_{1}$}

The intersection graph $\mathcal{I}(G)$ of all mis on $G$ is called the Independent Graph of $G$.

Theorem 5.1. The Independent Graph of $G_{n}=C_{n} \odot K_{1}$ is a complete 
graph with $\mu\left(G_{n}\right)$ vertices minus one edge if $n$ is even and it is a complete graph if $n$ is odd.

Proof. Let $V\left(C_{n}\right)=\left\{v_{1}, v_{2}, \ldots, v_{n}\right\}$ and $R=\left\{r_{1}, r_{2}, \ldots, r_{n}\right\}$ with $r_{i}$ the vertex of the i-th copy of $K_{1}$. Given an independent set $S$ of $C_{n}$, we have that $S \cup\left(R \backslash A d j_{R}(S)\right)$ is a mis of $G_{n}$. If $n$ is even, the only pair of disjoint mis in $G_{n}$ is $\left\{v_{1}, r_{2}, v_{3}, \ldots, r_{i}, v_{i+1}, r_{i+2}, \ldots, r_{n}\right\}$ and $\left\{r_{1}, v_{2}, r_{3}, \ldots, v_{i}, r_{i+1}\right.$, $\left.v_{i+2}, \ldots, v_{n}\right\}$. But if $n$ is odd there are no disjoint mis in $G_{n}$. In fact, since any mis of $G_{n}$ contains at most $\frac{n-1}{2}$ vertices of the cycle $C_{n}$, it must contain at least $\frac{n+1}{2}$ vertices of $R$. Thus, any pair of mis has at least one common vertex in $R$.

The Clique Graph $\mathcal{K}(G)$ of $G$ is the intersection graph of its cliques.

A chordless sun graph has vertex set $V\left(C_{n}\right) \cup V(R)$ such that $V\left(C_{n}\right)=$ $\left\{v_{1}, \ldots, v_{n}\right\}$ induces a chordless cycle $C_{n}$ and $R=\left\{r_{1}, \ldots, r_{n}\right\}$ is an independent set. Each vertex $r_{i}$ is adjacent to $v_{i}$ and $v_{(i m o d n)+1}$ for $i=1, \ldots, n$.

Theorem 5.2. For $n \geq 4$, the Clique Graph $\mathcal{K}\left(G_{n}\right)$ of the corona graph $G_{n}=C_{n} \odot K_{1}$ is a chordless sun graph $S\left(C_{n}\right)$.

Proof. Every clique of $G_{n}$ is isomorphic to $K_{2}$. Let $L_{i}$ be the clique induced by $\left\{v_{i}, v_{i+1}\right\}$ with $v_{i}, v_{i+1} \in V\left(C_{n}\right)$ for $i=1, \ldots, n-1$ and the clique $L_{n}$ induced by $\left\{v_{n}, v_{1}\right\}$. Call $M_{i}$ the clique induced by $v_{i}$ and $r_{i}$. $L_{1}$ shares $v_{1}$ with $M_{1}$ and with $L_{n}$. For $i=2, \ldots, n-1, L_{i}$ shares $v_{i}$ with $L_{i-1}$ and $M_{i}$. Moreover, $L_{i}$ shares $v_{i+1}$ with both $L_{i+1}$ and $M_{i+1}$. Thus, $G_{n}$ has $2 n$ cliques such that their associated vertices in $\mathcal{K}\left(G_{n}\right)$ induce a chordless cycle $C_{n}$ with $n$ triangles, each one sharing an edge with $C_{n}$. Triangle $T_{i}$ has vertices $L_{i-1}, L_{i}$ and $M_{i}, i=2, \ldots, n$ and $T_{1}$ has vertices $L_{1}, L_{n}$ and $M_{1}$.

\section{Conclusions}

We have determine the number of independent sets of various corona graphs. It can be deduced that $f_{k}\left(C_{n}\right)=f_{k}\left(P_{n}\right)-f_{k-2}\left(P_{n-1}\right)$. We obtain 
recursive and combinatorial expressions for the number of independent sets with a given size of a chordless path $P_{n}$ and a cycle $C_{n}$. It would be interesting to study the number of independent sets and to characterize the independent and the clique graph of other corona graphs. 


\section{References}

[1] G. J. Chang and M. J. Jou, The Number of Maximal Independent Sets in Connected Triangle-free Graphs, Discrete Mathematics 197198 (1999), 169-178.

[2] H. Deng and S. Chen, The Extremal Unicyclic Graphs with respect to Hosoya Index and Merrifield-Simmons Index, MATCH Commun. Math. Comput. Chem. 59 (2008), 171-190.

[3] S. El-Basil, Binomial-combinatorial Properties of Clar Structures, Discrete Applied Mathematics 19 (1998), no. 1-3, 145-156.

[4] R. Frucht and F. Harary, On the corona of two graphs, Aequationes Mathematicae 4 (1970), no.3, 322-325.

[5] Z. Füredi, The Number of Maximal Independent Sets in Connected Graphs, Journal of Graph Theory 11 (1987), 463-470.

[6] A. Hamzeh, A. Iranmanesh, S. Hossein-Zadeh and M. A. Hosseinzadeh, The Hosoya Index and the Merrifield-Simmons Index of Some Graphs, Transactions on Combinatorics 1 (2012), no.4, 51-60.

[7] M. Hujter and Z. Tuza. The Number of Maximal Independent Sets in Triangle-free Graphs, SIAM Journal on Discrete Mathematics 6(1993), 284-288.

[8] S. Li, H. Zhang and X. Zhang, Maximal Independent Sets in Bipartite Graphs with at least One Cycle, Discrete Mathematics and Theoretical Computer Science 15 (2013), no.2, 243-258.

[9] R. E. Merrifield and H. E. Simmons, Topological Methods in Chemistry (1989), Wiley, New York.

[10] Y. Okamoto, T. Uno and R. Uehara, Counting the Number of Independent Sets in Chordal Graphs, Journal of Discrete Algorithms 6 (2008), 229-242. 
[11] C. Ortiz and M. Villanueva, Maximal Independent Sets in Caterpillar Graphs, Discrete Applied Mathematics 160 (2012), 259-266.

[12] C. Ortiz and M. Villanueva, Maximal Independent Sets in Grid Graphs, International Transactions in Operational Research 24 (2007), 369-385.

[13] H. Prodinger and R. F. Tichy, Fibonacci Numbers of Graphs, Fibonacci Quart. 20 (1982), no.1, 16-21.

[14] M. H. Reyhani, S. Alikhani and M. A. Iranmanesh, Hosoya and Merrifield-Simmons Indices of Some Classes of Corona of Two Graphs, Transactions on Combinatorics 1 (2012), no.4, 1-7.

[15] L. G. Valiant, The Complexity of Computing the Permanent, Theoretical Computer Science 8 (1979), 189-201.

[16] S. Wu, J. Yang and H. Cheng, Merrifield-Simmons Index of the Corona of Two Graphs, South Asian Journal of Mathematics 2 (2012), no.3, 274-278.

[17] I. G. Yero and J. A. Rodriguez-VelÃ $i z q u e z$, On the Randic Index of Corona Product Graphs, ISRN Discrete Mathematics, Volume 2011, Article ID 262183, 7 pages. doi:10.5402/2011/262183.

Carmen Ortiz

Industrial Engineering School

Universidad de Valparaiso

Chile

carvic.ortiz@gmail.com
Mónica Villanueva

Informatic Engineering Department

Universidad de Santiago de Chile

Chile

monica.villanueva@usach.cl 